\title{
Importance of the kind of cue for judgments of learning (JOL) and the delayed-JOL effect
}

\author{
JOHN DUNLOSKY and THOMAS O. NELSON \\ University of Washington, Seattle, Washington
}

\begin{abstract}
The delayed-JOL effect is the finding in which judgments of learning (JOLs) are more accurate at predicting eventual recall when they are made a short time after study rather than immediately after study. The present research replicated this effect and found that the kind of cue that is used for JOLs is critical. In particular, following the study of stimulus-response paired associates, there is an extremely robust delayed-JOL effect when the cue for JOLs is the stimulus alone (every one of $\mathbf{4 5}$ subjects showed the effect); however, there is little, if any, delayed-JOL effect when the cue for JOLs is the stimulus-response pair. This finding has important implications for education: To have the greatest accuracy at predicting eventual recall, a person should make JOLs not immediately after study but, instead, shortly after study (i.e., delayed JOLs) with the cue for JOLs being the stimulus alone. The theoretical mechanisms for the delayed-JOL effect are currently unknown, but some speculations are offered.
\end{abstract}

Imagine two students studying French-English vocabulary (e.g., chateau-castle) for an upcoming examination. Both students self-monitor their studying by making a judgment of learning (JOL), which is a prediction of the likelihood of eventual memory performance for recently studied items, for each French-English pair. (For definitional distinctions between JOLs and other metamemory judgments, see Zechmeister \& Nyberg, 1982; for functional distinctions, see Leonesio \& Nelson, 1990; and for an overall theoretical framework that integrates the various kinds of metamemory judgments, see Nelson \& Narens, 1990.)

As the students study, their JOLs have a key role in how their study time is allocated. In particular, less study time is reallocated to information judged as easier to recall on a subsequent test (Mazzoni, Cornoldi, \& Marchitelli, 1990). Therefore, the accuracy of their JOLs at predicting eventual recall is critical for the efficiency of self-paced study. For instance, if accuracy is low, more study might be allocated to items well learned than to items poorly learned - thus potentially resulting in slower learning and/or lower eventual memory performance.

One example of how monitoring accuracy is critical for the efficiency of study comes from Bisanz, Vesonder, and Voss (1978). During multitrial learning, college students studied and then were tested on a list of paired associates; these study-test trials were repeated until one errorless trial was achieved. Before restudying an item, the students monitored whether they had correctly recalled

This research was supported by NIMH Grant MH32205. We thank Hunter Hoffman and Björn Levidow for comments on a preliminary draft, Ian Begg and Robert Bjork for comments on the submitted manuscript, and Tiffany Olsen for help in collecting the data. Requests for reprints should be sent to either author at the University of Washington, Psychology NI-25, Seattle, WA 98195. or had not correctly recalled the item on the immediately previous test trial. Most important, the students with the greatest monitoring accuracy needed the fewest study trials to achieve one errorless test trial.

Similarly, other researchers have found that (1) students with the greatest monitoring accuracy also have the greatest test performance (Maki \& Berry, 1984) and (2) more study time is allocated to difficult items than to easy items by expert than by novice memorizers (IntonsPeterson \& Smyth, 1987). Both of these relationships involving increased monitoring accuracy-that is, with increased test performance and with the allocation of extra study time to the more difficult items-were reported in a single experiment by Owings, Petersen, Bransford, Morris, and Stein (1980). Because of the role that metacognitive monitoring can play in learning and memory, we investigated how students such as the two mentioned in the opening paragraph should make JOLs so as to increase the accuracy of their JOLs.

\section{The Delayed-JOL Effect}

One way the two students could make JOLs is to conceal the response (e.g., by covering castle with the hand) so as to use the stimulus alone (e.g., chateau-?) as a cue for the judgments. One student makes a JOL for an item immediately after studying it (called an immediate JOL). In contrast, the other student studies an item and then waits a short time (e.g., $30 \mathrm{sec}$ of studying other items) before making a JOL for the item (called a delayed JOL, because there is a delay between the study and JOL for a given item). Which student's JOLs will be more accurate in predicting eventual recall performance?

In accord with the delayed-JOL effect (Nelson \& Dunlosky, 1991), the student making delayed JOLs should be more accurate. This effect refers to the finding that when the cue for JOLs is the stimulus alone, the accuracy of 
JOLs in predicting eventual recall is greater for delayed JOLs than for immediate JOLs. Moreover, the size of the delayed-JOL effect is remarkable. In Nelson and Dunlosky (1991), the average Goodman-Kruskal gamma correlation $(G)$ between JOLs and recall was +.38 for $\mathrm{im}$ mediate JOLs, but was close to perfect $(G=+.90)$ for delayed JOLs. ${ }^{1}$ The delayed-JOL effect has important implications for education. Namely, to best predict eventual recall, a person should make JOLs shortly after study instead of immediately after study.

\section{Does the Delayed-JOL Effect Occur When JOLs Are Cued by the Stimulus-Response Pair?}

Although students making delayed JOLs cued by the stimulus alone would achieve a large boost in JOL accuracy, another potentially reasonable way in which the two students might make JOLs (instead of concealing the response and using the stimulus alone as a cue) would be to use both the stimulus and response as a cue (e.g., chateau-castle) for their judgments. Then, if one student should make immediate JOLs, and the other, delayed JOLs, which student's judgments would be more accurate at predicting eventual recall? Put another way, will the delayedJOL effect occur when the cue for JOLs is the stimulusresponse pair? And which of the four conditions (defined by immediate versus delayed JOLs and by stimulus-alone vs. stimulus-response cues) yields the greatest accuracy at predicting eventual recall?

The answers to both questions will have important implications for how people should monitor their own learning. Regarding the first question, if the delayed-JOL effect occurs when JOLs are cued by the stimulus-response pair, then one should use delayed JOLs instead of immediate JOLs when using the stimulus-response pair as a cue. Regarding the second question, if accuracy is greatest for delayed JOLs cued by the stimulus alone, then this kind of JOL should be used to self-monitor learning. Alternatively, if two or more conditions are tied in yielding greatest accuracy, then how one makes JOLs could be based on another criterion (e.g., use the kind of JOL that yields both the greatest JOL accuracy and the greatest eventual recall performance after multitrial learning).

In the present experiment, college students studied stimulus-response pairs and made either an immediate or delayed JOL for each pair. The kind of cue for JOLs was either the stimulus-response pair or the stimulus alone.

\section{METHOD}

\section{Materials}

The items were 66 concrete ( $C \geq 6.08$; Paivio, Yuille, \& Madigan, 1968, norms), unrelated, noun-noun paired associates (e.g. ocean-tree). Apple II computers displayed instructions and items and recorded all responses.

\section{Design}

The interval between the study and JOL of an item (immediate or delayed JOLs, as described below) was a within-subject manipulation. The kind of cue for the JOLs (either the stimulus alone or the stimulus and response of an item) was a between-subject manipulation.

\section{Subjects}

One hundred undergraduates from the University of Washington participated to receive extra credit. Fifty subjects were randomly assigned to each group by order of appearance. The subjects participated individually.

\section{Procedure}

The task included one paired-associate study trial; items were presented in a random order for $6 \mathrm{sec} / \mathrm{item}$. A subject-paced JOL was made for every item, and the JOL was cued with either the stimulus alone (c.g., ocean-_..... ?) or the stimulus-response pair (e.g., ocean-tree), and the query, "How confident are you that in about $10 \mathrm{~min}$ from now you will be able to recall the second word of the item when prompted with the first?" $(0=$ definitely will not recall, $20=20 \%$ sure, $40=40 \%$ sure, $60=60 \%$ sure, $80=$ $80 \%$ sure, and $100=$ definitely will recall).

List construction. The first six items (primacy buffer) served as practice for study and were not included in the criterion task. The remaining items comprised two blocks of 30 items/block. Each item was randomly slated for an immediate or delayed JOL, with the only restrictions being that 15 items in each block were slated for each kind of JOL and no more than 3 consecutive items were slated for the same kind of JOL.

Each immediate JOL occurred immediately after the offset of an item. The delayed JOLs of a given block occurred as follows: After the study trial or final immediate JOL of the block, the JOLs for the first third of items presented during study that were slated for delayed JOLs occurred in random order; then the JOLs for the second third of items occurred in random order, and so forth. This ensured that there were at least 10 other items (being studied or receiving JOLs) between the study and JOL for every item slated for a delayed JOL.

Paired-associate recall. Following the final delayed JOL of the second block, there was an unrelated filler activity for $10 \mathrm{~min}$ (pilot data showed that a 10-min retention interval and 6-sec/item presentation rate led to intermediate recall performance), followed by paired-associate recall. Recall trials were subject paced, and the subjects could not omit a response. The order of items for recall was determined as follows: The first 10 items presented during study were randomized anew and presented for recall; then the second 10 items presented during study were randomized anew for recall; and so forth.

For recall trials, the stimulus was presented and the subjects were asked to type the second word of the item. To minimize the role of incorrect spelling, if the first three letters of an answer were correct, it was scored as correct. Prior to the experiment, the subjects were instructed to work hard to learn the items and were informed about the eventual recall tests.

\section{RESULTS}

\section{JOL Accuracy}

Both relative JOL accuracy and absolute JOL accuracy were evaluated as in Nelson and Dunlosky (1991). Relative JOL accuracy is how well a person predicts the likelihood of correct recall for one item relative to another item. For example, with relative accuracy, we are interested in whether an item given a higher JOL (e.g., of $80 \%$ ) has a greater probability of correct recall than does an item given a lower JOL (e.g., of $40 \%$ ). Moreover, relative JOL accuracy is most appropriate when a person must decide whether to allocate an extra re-study trial to one item as opposed to another item (e.g., when deciding to re-study cheval-horse vs. chateau-castle). By contrast, absolute JOL accuracy is how well a person predicts the 
likelihood of correct recall for a particular set of items. For example, with absolute JOL accuracy, we are interested in the actual percentage of correct recall for items given a particular JOL (i.e., the predicted percentage of correct recall), and most important, how close the predicted percentage is to the actual percentage of correct recall.

\section{Relative JOL Accuracy}

The relative accuracy of JOLs in predicting eventual recall was operationalized via a Goodman-Kruskal gamma correlation between JOLs and recall. For each subject, one gamma was calculated for items that had immediate JOLs and another was calculated for items that had delayed JOLs ( 5 subjects cued by the stimulus alone and 6 subjects cued by the stimulus-response pair were excluded from the analyses of relative JOL accuracy because of indeterminate gammas). The cumulative percentage of people attaining a given level (or greater) of relative JOL accuracy for each of the four conditions (defined by immediate vs. delayed JOLs and by the stimulus-alone vs. stimulus-response cues) is plotted in Figure 1, where distinctions are made until almost all of the subjects having delayed stimulus-alone cues are accounted for.

The kind of cue for JOLs and the delayed-JOL effect. In accord with previous findings (Nelson \& Dunlosky, 1991), when JOLs were cued by the stimulus alone, relative JOL accuracy was much greater for delayed JOLs

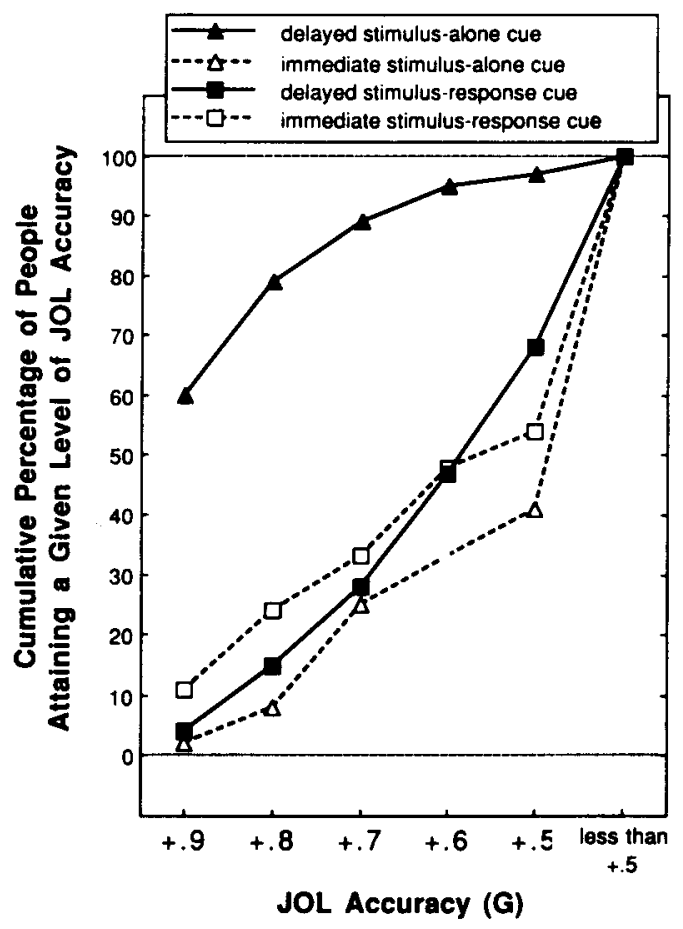

Figure 1. The cumulative percentage of people attaining a given level (or higher) of relative JOL accuracy, showing the advantage for people whose JOLs were delayed and cued by the stimulus alone (vs. three other conditions). (median $\mathrm{G}=+.93$ ) than for immediate JOLs (median $\mathbf{G}=+.45$ ). The difference between these two conditions was highly reliable $(p<.001$, by a sign test), and relative JOL accuracy was greater for delayed than for immediate JOLs for every one of the 45 subjects whose JOLs were cued by the stimulus alone!

When JOLs were cued by the stimulus-response pair, relative $\mathrm{JOL}$ accuracy was not reliably different $(p=.18$, by a sign test) for delayed JOLs (median $G=+.60$ ) than for immediate JOLs (median $G=+.55$ ). Thus, the delayed-JOL effect is extraordinarily robust when JOLs are cued by the stimulus alone, but there is little, if any, delayed-JOL effect when JOLs are cued by the stimulusresponse pair. ${ }^{2}$

Which condition yielded the greatest relative JOL accuracy? As shown in Figure 1, relative JOL accuracy was greater for delayed JOLs cued by the stimulus alone than for (1) immediate JOLs cued by the stimulus alone (see analysis above), (2) immediate JOLs cued by the stimulus-response pair (Mann-Whitney $U$ test $=1,464$, $p<.001$ ), and (3) delayed JOLs cued by the stimulusresponse pair (Mann-Whitney $U$ test $=1,424, p<$ $.001)$. (The third finding replicated a finding in Begg, Duft, Lalonde, Melnick, \& Sanvito, 1989, Experiment 4; their experiment did not include a comparison between immediate vs. delayed JOLs.) Moreover, more than 60\% of the subjects using delayed JOLs cued by the stimulus alone attained a relative JOL accuracy of at least +.90 , whereas fewer than $20 \%$ of the subjects from each of the other conditions attained that level of relative JOL accuracy (see Figure 1).

\section{Absolute JOL Accuracy}

Absolute JOL accuracy was operationalized via calibration curves. One calibration curve was constructed for each of the four conditions (defined by immediate vs. delayed JOLs and by the stimulus-alone vs. stimulusresponse cues) as follows: Items given the same predicted percentage of correct recall by a given subject were aggregated, the actual performance of correct recall was calculated for that aggregate, and the mean (across subjects) actual percentage of correct recall was plotted against the predicted percentage of correct recall. The calibration curves and the line of perfect calibration (shown as the dotted line comprising the main diagonal) are shown in Figures 2 and 3.

The kind of cue for JOLs and the delayed-JOL effect. The calibration curves for JOLs cued by the stimulus alone are shown in the top panel of Figure 2. In accord with previous research (Nelson \& Dunlosky, 1991), the calibration curve is closer to the main diagonal of perfect calibration for delayed than for immediate JOLs. ${ }^{3}$ By contrast, for JOLs cued by the stimulus-response pair (bottom panel of Figure 2), neither the calibration curve for immediate JOLs nor the calibration curve for delayed JOLs is systematically closer to the main diagonal of perfect calibration. ${ }^{4}$ Thus, the results for absolute and relative JOL accuracy were consistent in confirming the same 

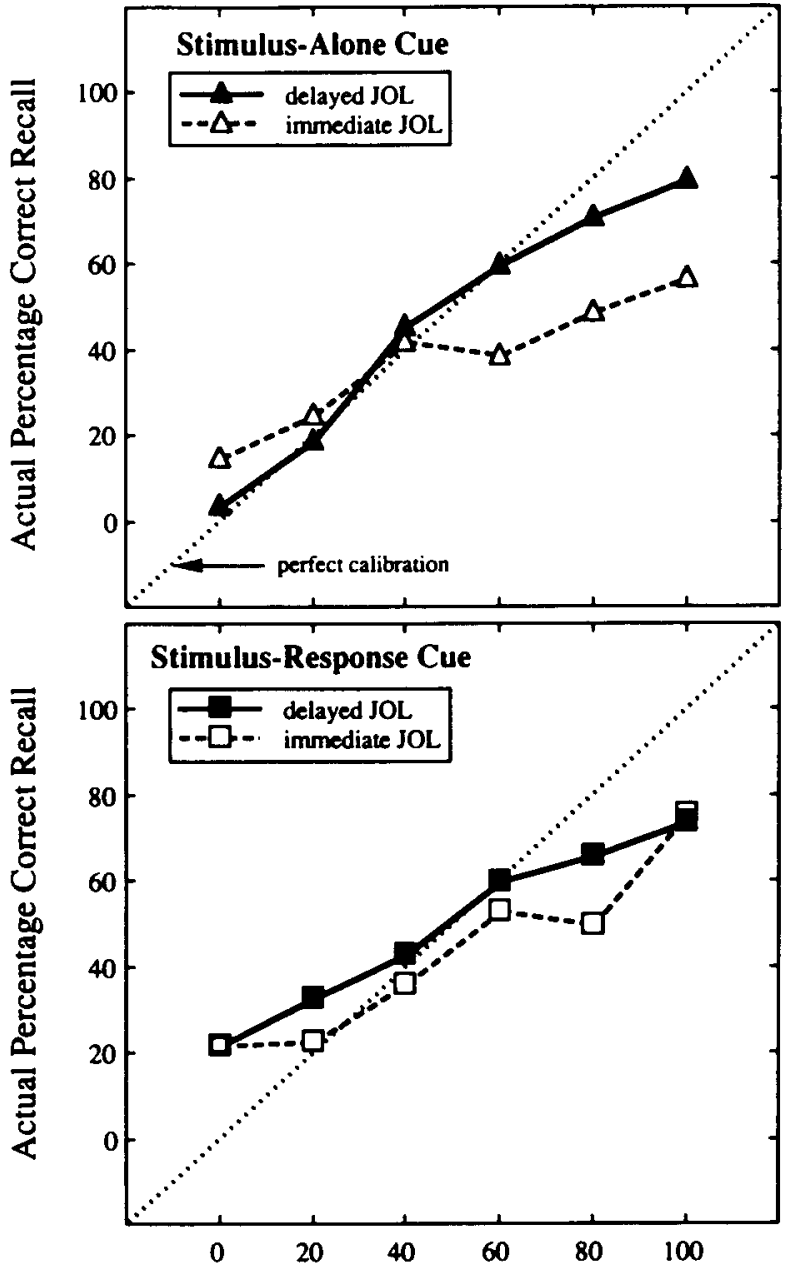

Predicted Percentage Correct Recall

Figure 2. The mean percentage of correct recall at each level of predicted recall for immediate JOLs and delayed JOLs. For JOLs cued by the stimulus alone (top panel), the delayed JOLs were more accurate than the immediate JOLs; however, no systematic difference-in terms of closeness to the main diagonal of perfect calibration accuracy-occurred when the delayed versus immediate JOLs were cued by the stimulus-response pair (bottom panel).

conclusion: The delayed-JOL effect is present when JOLs are cued by the stimulus alone but is absent when JOLs are cued by the stimulus-response pair.

Which condition yielded the greatest absolute JOL accuracy? By examining the top panel in Figure 2 and the two panels in Figure 3, the reader can see that the calibration curve is closer to the main diagonal of perfect calibration for delayed JOLs cued by the stimulus alone than for any of the other three conditions: (1) immediate JOLs cued by the stimulus-response pair (top panel of Figure 3, discussed earlier), (2) immediate JOLs cued by the stimulus alone (top panel of Figure 2), and (3) delayed JOLs cued by the stimulus-response pair (bottom panel of Fig- ure 3). The parameter values of lines of best fit (see Notes 3 and 4) also confirm that conclusion. Thus, absolute accuracy, as well as relative accuracy (Figure 1), is better for delayed JOLs cued by the stimulus alone than for the other three conditions.

Does the number of different JOL ratings used by subjects play a role in the superior accuracy of delayed stimulus-alone JOLs? For each of the four conditions, the frequency distribution of subjects who used various numbers of different JOL ratings is reported in Table 1 . These distributions were useful for evaluating the possibility that the number of different JOL ratings used by the subjects is somehow responsible for the aforementioned conclusions about JOL accuracy.

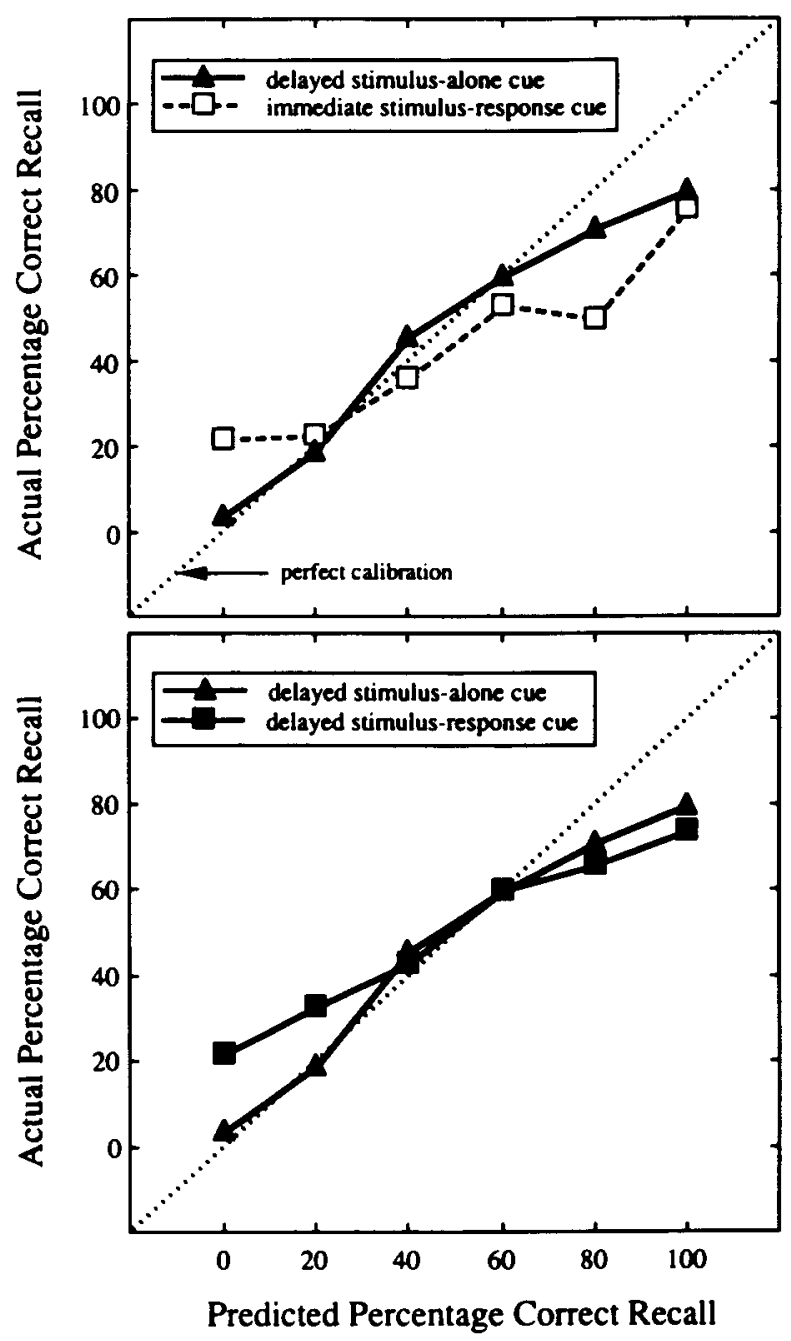

Figure 3. The mean percentage of correct recall at each level of predicted recall. The delayed JOLs cued by the stimulus alone were more accurate thm the immedinte JOLs cued by the stimulus-response pair (top panel) and were more accurate than the delayed JOLs cued by the stimulus-response pair (bottom panel). 
Table 1

Frequency of Subjects who Used Various Numbers of Different JOL Ratings

\begin{tabular}{|c|c|c|c|c|c|}
\hline \multirow[b]{2}{*}{ Condition } & \multicolumn{5}{|c|}{ Number of Different JOL Ratings Used } \\
\hline & 1 & 2 & 3 & 4 & 5 \\
\hline \multicolumn{6}{|c|}{ Stimulus-Alone Cue } \\
\hline Delayed JOL & 0 & 4 & 13 & 13 & 8 \\
\hline Immediate JOL & 0 & 1 & 8 & 17 & 14 \\
\hline \multicolumn{6}{|c|}{ Stimulus-Response Cue } \\
\hline Delayed JOL & 1 & 7 & 6 & 13 & 14 \\
\hline Immediate JOL & 0 & 1 & 8 & 20 & 14 \\
\hline
\end{tabular}

Specifically, the possibility (suggested by a reviewer) was that the delayed JOLs cued by the stimulus alone may have yielded near-perfect accuracy because each subject may have used only two different JOL ratings for his or her delayed stimulus-alone JOL ratings. However, as is evident from the first row of Table 1, the present data are inconsistent with this explanation: 46 out of 50 (i.e., $92 \%$ ) of the subjects who had delayed stimulus-alone JOL cues used three or more JOL ratings. Thus, the nearperfect accuracy of delayed stimulus-alone JOLs cannot be accounted for by a hypothesis based on the assumption that only two different JOL ratings were used for that condition and/or that there was accurate monitoring of merely a few recallable items. This finding, together with the fact that the calibration curve for delayed JOLs cued by the stimulus alone is close to the line of perfect calibration (Figure 2), disconfirms such a hypothesis and instead confirms the idea that there is finer-than-binary differentiation between the items during delayed stimulus-alone JOLs.

Another possibility related to the one suggested above is that greater JOL accuracy occurred for the delayed stimulus-alone JOLs than for any of the other three conditions because the number of different JOL ratings was not the same for subjects in the delayed stimulus-alone JOL condition as it was in the other conditions. However, as indicated in Table 1 , the number of different JOL ratings used by subjects does not differ significantly across conditions $\left[\chi^{2}(15)=20.45, p>.10\right]$, and it therefore could not have been responsible for the aforementioned differences in JOL accuracy.

\section{Paired-Associate Recall}

Although recall performance after a single pairedassociate study trial may have only little relevance to a naturalistic setting in which students use multiple-study trials, the present recall performance was analyzed because it was used in the analyses of JOL accuracy. For each subject, the proportion of correct recall was determined for items that previously had intermediate JOLs and for items that previously had delayed JOLs. A $2 \times 2$ analysis of variance was conducted on those proportions to assess the effects of the interval between study and JOL (immediate vs. delayed JOL) and the kind of cue for JOLs (stimulus-alone vs. stimulus-response cue).
The present procedure yielded intermediate recall performance (which made the previously discussed analyses of JOL accuracy possible): the overall mean proportion of recall performance across all conditions was .35. Although the means from the conditions did not deviate by more than .07 from the overall mean, there were statistically reliable differences between conditions. There was a main effect for JOL interval $\left[F(1,98)=9.72, M S_{\mathrm{e}}=\right.$ $.013, p<.01]$, but not for the kind of cue for JOLs $\left[F(1,98)=1.88, M S_{\mathrm{e}}=.094, p=.17\right]$. However, the interaction of JOL interval $\times$ cue was reliable $[F(1,98)=$ 23.61, $\left.M S_{\mathrm{c}}=.013, p<.001\right]$. Recall for items that had delayed JOLs cued by the stimulus-response pair $(M=$ $.44, S E M=.04$ ) was greater than recall for the corresponding pairs that had immediate JOLs $[M=.32$, $S E M=.03, t(49)=5.49, p<.001]$. By contrast, for items that had JOLs cued by the stimulus alone, the difference between immediate $(M=.33, S E M=.03)$ and delayed $(M=.31, S E M=.03)$ was not reliable $[t(49)=$ $1.27, p=.21$ ]. Recall for items that had delayed JOLs cued by the stimulus-response was also greater than recall for items that had immediate JOLs cued by the stimulus alone $[t(98)=2.26, p<.05]$ and that had delayed JOLs cued by the stimulus alone $[t(98)=2.74, p<.01]$.

\section{DISCUSSION}

\section{Educational Implications}

The cue for JOLs is critical for the delayed-JOL effect. The present research replicated the delayed-JOL effect (Nelson \& Dunlosky, 1991), which is the increase in JOL accuracy when JOLs are made shortly after study instead of immediately after study. A new finding was that the cue for JOLs is critical: When the cue is the stimulus alone, the delayed-JOL effect is extremely robust, but when the cue is the stimulus-response pair, the delayedJOL effect is negligible. Moreover, accuracy was greatest for delayed JOLs cued by the stimulus alone--both for relative JOL accuracy (Figure 1) and for absolute JOL accuracy (top panel of Figure 2 and both panels of Figure 3)-than for any of the other three conditions.

Therefore, to achieve the greatest accuracy at predicting eventual recall, people should monitor their learning by (1) making JOLs shortly after study instead of immediately after study, and (2) cuing the JOLs with the stimulus alone. One strategy that could be used to make such JOLs while learning a list of foreign-language vocabulary is the following: An item (e.g., chateau-castle) is studied, and before the JOL for the item is made, at least $30 \mathrm{sec}$ should be spent on something else (e.g., studying other items). Then, a JOL is made for chateau-castle by concealing castle and answering the query, "How likely am I to recall the English translation of chateau at a later time?" The strategy would be continued until every item was studied and judged.

As suggested by the present research, most people using this strategy should have near-perfect JOL accuracy (Fig- 
ure 1). Moreover, this strategy should help in reallocating subsequent study time more efficiently, because people could more accurately isolate (for re-study) the items that are less well learned.

The kind of cue for JOL and recall. Although not directly relevant to the findings concerning JOL accuracy, the two kinds of cues for delayed JOLs had different effects on JOL accuracy as opposed to recall. Namely, delayed JOLs cued by the stimulus alone yielded much greater JOL accuracy than did delayed JOLs cued by the stimulus-response (Figure 1), whereas recall was somewhat greater for delayed JOLs cued by the stimulus-response pair than for delayed JOLs cued by the stimulus alone.

Bahrick and Hall's (1991) concepts of preventive maintenance and corrective maintenance provide one explanation for the superior recall of items with delayed JOLs cued by the stimulus-response pair. Preventive maintenance occurs when an intervention (e.g., a cue for JOL, an additional study or recall trial, etc.) prevents the loss of access to a recallable item, whereas corrective maintenance occurs when an intervention reestablishes lost access to a nonrecalled item. Thus, items having delayed stimulus-response JOLs can potentially receive either preventive or corrective maintenance. By contrast, items having delayed stimulus-alone JOLs can receive only preventive maintenance (e.g., if the stimulus-alone cue elicits the correct response) and cannot receive corrective maintenance. The delayed stimulus-alone JOLs should provide negligible facilitation of subsequent recall for responses that are not retrieved at the time of the JOL (cf. Modigliani, 1976).

Because eventual recall is typically greater when preventive maintenance occurs via an extra test trial than when it occurs via an extra study trial (Bjork, 1988; Modigliani, 1976; Runquist, 1983), overall recall of the list could conceivably be greater after delayed stimulusalone JOLs rather than after delayed stimulus-response JOLs, for example, when many items receive preventive maintenance and few items require corrective maintenance. By contrast, when there are relatively fewer instances of the stimulus-alone cue eliciting the correct response, eventual recall should be greater for items with delayed JOLs cued by a stimulus-response pair than cued by the stimulus alone (as in the present experiment).

Are there conditions that will potentially optimize JOL accuracy and recall? An important goal for future research should be to isolate conditions that optimize both $\mathrm{JOL}$ accuracy and recall. One possibility would be to use a hybrid cue to make JOLs. For instance, when the correct response cannot be retrieved during a delayed JOL cued by the stimulus alone (e.g. chateau-___? the response could be prompted by revealing its first letter (e.g. chateau-c_? , and if still unsuccessful, then by revealing the first two letters (e.g., chateau-ca__?), and so forth, until the response is eventually retrieved (Gruneberg \& Monks, 1974). Therefore, for a single study trial wherein the delayed JOL is initially cued by the stimulus alone, the entire stimulus-response pair would always oc- cur (which resembles a stimulus-response cue), thereby facilitating subsequent recall. Future research should investigate the effect of such hybrid cues on JOL accuracy and recall (both in terms of single-trial and multitrial learning).

\section{Implications for Theories of Metamemory}

Besides having educational implications, the present findings also have implications for possible mechanisms of the metamemory system. Any complete theory of metamemory should explain both why JOL accuracy is greater for delayed JOLs than for immediate JOLs when the JOLs are cued by the stimulus alone, and why JOL accuracy is no greater for delayed JOLs than for immediate JOLs when the JOLs are cued by the stimulus-response pair.

One explanation for this pattern of findings is that when people assess the likelihood of eventual recall for recently studied information, they may simultaneously monitor both short-term and long-term memory (referred to as the monitoring-dual-memories explanation; Nelson \& Dunlosky, 1991). This explanation suggests that for immediate JOLs, information about the stimulus-response pair in short-term memory adds noise to or dominates the monitoring (i.e., retrieval) of information in long-term memory. This reduces the accuracy of immediate JOLs because eventual recall will be based on information only in long-term memory. By contrast, delayed JOLs exceed the span of retrieval from short-term memory (i.e., less than $30 \mathrm{sec}$, Peterson \& Peterson, 1959) and thereby allow better interrogation of long-term memory via retrieval of the information contained therein, without noise from information about that item in short-term memory. However, in the case of delayed JOLs cued by the stimulusresponse pair, the stimulus-response may be attended to (e.g., entered into short-term memory and then retrieved) before the person can retrieve the information from longterm memory about that item (see the latencies in Wescourt \& Atkinson, 1973). This information from short-term memory about the item would produce the same kind of monitoring problems as those which occur in the case of immediate JOLs.

Another explanation can be derived from the notion of transfer-appropriate monitoring: As the similarity increases between the context of monitoring and the context of eventual retrieval, the accuracy of monitoring will increase (cf. Begg et al., 1989). In the present research, the delayed JOLs cued by the stimulus alone were the most similar to the eventual recall task. A testable prediction is that when the criterion task is the recognition of stimulus-response pairs, ${ }^{5} \mathrm{JOL}$ accuracy should be greater for delayed JOLs that are cued by the stimulus-response pair (which are more similar to the context of the recognition of the stimulus-response pairs) than for delayed JOLs cued by the stimulus alone.

Future research should empirically investigate those and other explanations. ${ }^{6}$ However, regardless of what the best explanation of the delayed-JOL effect is, the present findings will be important for applied situations (as elaborated 
above). In particular, when presented with the choice of whether to use immediate or delayed JOLs, and whether to cue the JOLs by the stimulus alone or by the stimulusresponse pair, a person who wants to be most accurate at predicting the likelihood of eventual recall performance should make delayed JOLs that are cued by the stimulus alone.

\section{REFERENCES}

BAhrick, H. P., \& HALl, L. K. (1991). Preventive and corrective maintenance of access to knowledge. Applied Cognitive Psychology, $5,1-18$.

Begg, I., Duft, S., Lalonde, P., Melnick, R., \& Sanvito, J. (1989). Memory predictions are based on ease of processing. Joumal of Memory \& Language, 28, 610-632.

Bisanz, G. L., Vesonder, G. T., \& Voss, J. F. (1978). Knowledge of one's own responding and the relation of such knowledge to learning. Journal of Experimental Child Psychology, 25, 116-128.

BJoRk, R. A. (1988). Retrieval practice and the maintenance of knowledge. In M. M. Gruneberg, P. Morris, \& R. N. Sykes, (Eds.), Practical aspects of memory: Current research and issues (Vol. 1, pp. 396401). New York: Wiley.

Grunererg, M. M., Monks, J. (1974). Feeling of knowing and cued recall. Acta Psychologica, 38, 257-265.

Intons-Peterson, M. J., \& SMYTH, M. M. (1987). The anatomy of repertory memory. Joumal of Experimental Psychology: Learning, Memory, \& Cognition, 13, 490-500.

JACOBY, L. L., KELLEY, C. M. (1987). Unconscious influences of memory for a prior event. Personality and Social Psychology Bulletin, 13, 314-336.

Leonesio, R. J., Nelson, T. O. (1990). Do different measures of metamemory tap the same underlying aspects of memory? Journal of Experimental Psychology: Learning, Memory, \& Cognition, 16. 464-470.

MAKI, R. H., \& BERRY, S. L. (1984). Metacomprehension of text material. Joumal of Experimental Psychology: Learning, Memory, \& Cognition, 10, 663-679.

Mazzoni, G., Cornoldi, C., \& Marchitelli, G. (1990). Do memorability ratings affect study-time allocation? Memory \& Cognition, 18, 196-204.

Modigliani, V. (1976). Effects on a later recall by delaying initial recall. Journal of Experimental Psychology: Human Learning \& Memory, 2, 609-622.

Nelson, T. O. (1984). A comparison of current measures of the accuracy of feeling-of-knowing predictions. Psychological Bulletin, 95, 109-133.

Nelson, T. O., Dunlosky, J. (1991). When people's judgments of learning (JOLs) are extremely accurate at predicting subsequent recall: The "delayed-JOL effect." Psychological Science, 2, 267-270.

Nelson, T. O., Narens, L. (1990). Metamemory: A theoretical framework and new findings. In G. H. Bower (Ed.), The psychology of learning and motivation (Vol. 26, pp. 125-173). New York: Academic Press.

Owings, R. A., Petersen, G. A., Bransford, J. D., Morris, C. D., * STEIN, B. S. (1980). Spontaneous monitoring and regulation of learning: A comparison of successful and less successful fifth graders. Journal of Educational Psychology, 72, 250-256.

Paivio, A., Yuille, J. C., \& Madigan, S. A. (1968). Concreteness, imagery, and meaningfulness values for 925 nouns. Journal of Experimental Psychology Monographs, 76(1, Pt. 2).

Peterson, L. R., Peterson, M. J. (1959). Short-term retention of individual verbal items. Joumal of Experimental Psychology, 58, 193-198.

Runquist, W. N. (1983). Some effects of remembering on forgetting. Memory \& Cognition, 11, 641-650.

Wescourt, K. T., \& AtKinson, R. C. (1973). Scanning for informa- tion in long- and short-term memory. Journal of Experimental Psychology, 98, 95-101.

Zechmeister, E. B., \& Nyerg, S. E. (1982). Human memory: An introduction to research and theory. Monterey, CA: Brooks/Cole.

\section{NOTES}

1. G has a range from -1.0 to +1.0 , with 0 representing nil accuracy and +1.0 representing perfect accuracy, and it is the best of the available measures for summarizing the accuracy of metamemory monitoring judgments (for reasons, see Nelson, 1984). Also, $G$ has a probabilistic interpretation, which, when applied to the accuracy of JOLs, is as follows: If a person gives one item a higher JOL than another item, and one of those two items is eventually recalled, whereas the other is not, then what is the probability $(p)$ that the recalled item was the one that had originally received the higher JOL? Thus, $p=.50$ when the JOLs are completely uninformative (i.e., nil accuracy, as if the JOLs were assigned by chance), and $p=1.0$ when the JOLs are perfectly accurate. Most important is that we can determine $p$ from $\mathrm{G}$ via the equation $p=$ $.5+.5 \mathrm{G}$ (derived in Nelson, 1984, Equation 7). Accordingly, in Nelson and Dunlosky (1991), $p=.95$ for delayed JOLs and .69 for immediate JOLs. Put differently, immediate JOLs cued by the stimulus alone were only $19 \%$ above chance at discriminating between recalled versus unrecalled items; by contrast, delayed JOLs cued by the stimulus alone were only $5 \%$ away from perfect accuracy!

2. Previously unreported findings from another experiment conducted in our laboratory were consistent with this null result. There was even a nonreliable trend for the magnitude of JOL accuracy to be greater for immediate than for delayed JOLs when cued by the stimulus-response pair.

3. Absolute JOL accuracy can also be evaluated by comparing the parameters from the least squares linear equations of best fit (that are fit to the data points of the calibration curves) to the corresponding parameters of the line of perfect calibration (equation: $y=0.0+1.0 x$. with an intercept of 0.0 and a slope of +1.0 , where $y$ is the actual percentage of correct recall and $x$ is the predicted percentage of correct recall). Confirming the conclusions in the text, the parameter values for the delayed stimulus-alone cue $(y=7.4+0.79 x)$ were closer to perfect calibration than were the corresponding parameter values for the immediate stimulus-alone cue $(y=17.9+0.40 x)$.

4. The parameter values for JOLs cued by the stimulus-response pair yielded no systematic advantage for either condition: for immediate JOLs, $y=17.0+0.53 x$, and for delayed JOLs, $y=22.4+0.58 x$. Especially notice the near-identical slopes of those lines of best fit.

5. It is important to distinguish between recognition of the stimulusresponse pair (e.g., chateau-castle) versus recognition of either the stimulus alone (chateau) or the response alone (castle) because the former is what is most crucial for people during the acquisition of foreignlanguage vocabulary - not the fact that chateau was one of the French words they were supposed to learn a response to, and not the fact that castle was one of the English words they were supposed to associate to a French word.

6. Two other explanations for why delayed stimulus-response JOLs are less accurate than delayed stimulus-alone JOLs were suggested during the editorial review. The reviewer, R. Bjork, suggested that an explanation might be derived from "subjective experience versus theory as a basis for judgments" (as discussed in Jacoby \& Kelley, 1987). The editor, S. Dumais, suggested that JOL accuracy may be lower for delayed stimulus-response JOLs than for delayed stimulus-alone JOLs because (1) the former may produce extra learning for some of the weaker items (namely, items that had received lower JOLs) and (2) this extra learning will somehow increase their likelihood of being recalled beyond that of items that had received higher JOLs. It would be interesting to see these two explanations tested by empirical investigations involving JOLs.

(Manuscript received May 2, 1991; revision accepted for publication December 31, 1991.) 